\title{
Mediação Parental na Exposição às Redes Sociais e a Internet de Crianças e
}

\author{
Adolescentes \\ Fernanda Tabasnik Schwartz* \\ Universidade Federal de Ciências da Saúde de Porto Alegre - UFCSPA, Porto Alegre, RS, Brasil \\ ORCID: https://orcid.org/0000-0001-5438-6437 \\ Janaína Thais Barbosa Pacheco** \\ Universidade Federal de Ciências da Saúde de Porto Alegre - UFCSPA, Porto Alegre, RS, Brasil \\ ORCID: https://orcid.org/0000-0001-5608-7457
}

\begin{abstract}
RESUMO
A exposição às redes sociais e a internet tem crescido de forma significativa, sendo cada vez mais frequente em crianças e adolescentes para diversas finalidades, representando um desafio para a parentalidade. As estratégias que os pais utilizam para lidar com esse comportamento podem se constituir em fatores de risco ou de proteção. Considerando os desafios comumente enfrentados pelos pais a fim de monitorar os filhos e protegê-los de determinados riscos relacionados à exposição às novas tecnologias, este artigo teórico objetivou descrever e discutir o uso das redes sociais e da internet na infância e na adolescência, assim como o impacto deste na parentalidade. A partir da revisão realizada, observou-se que os estudos sobre o tema indicam a importância da mediação e do monitoramento parental sobre a exposição de crianças e adolescentes às redes sociais e internet, resultando em um fator de proteção ao desenvolvimento. Além disso, observou-se que pais e filhos podem divergir com relação à percepção do controle parental exercido.
\end{abstract}

Palavras-chave: mídias sociais, videogames, estilos parentais, infância, adolescência.

\section{Parental Mediation in the Exposure to Social Networks and Internet of}

\section{Children and Adolescents}

\begin{abstract}
Exposure to social networks and the internet has grown significantly, being more frequent among children and adolescents for different purposes and representing a challenge for parenting. The strategies that parents use to deal with this behavior can constitute risk or protective factors. Considering the challenges commonly faced by parents in order to monitor and protect their children from certain risks, this theoretical article aimed to describe and discuss the use of social networks and internet in childhood and adolescence, as well as the impact of this use on parenting. From the review carried out, it was observed that studies on the subject indicate out the importance of mediation and parental monitoring on the exposure
\end{abstract}

ISSN $1808-4281$ 
of children and adolescents to social networks and electronic games, resulting in a protective factor for development. In addition, it was observed that parents and children may differ regarding the perception of parental control exercised.

Keywords: social media, games, parenting style, childhood, adolescence.

\section{Mediación Parental en la Exposición a Redes Sociales e Internet de Niños y}

\section{Adolescentes}

\section{RESUMEN}

La exposición a las redes sociales e internet ha crecido significativamente, siendo cada vez más frecuente en niños y adolescentes con diferentes propósitos y representando un desafío para la crianza de los hijos. Las estrategias que los padres usan para lidiar con este comportamiento pueden constituir factores de riesgo o de protección. Este artículo teórico tuvo como objetivo describir y discutir el uso de las redes sociales y la internet en la infancia y la adolescencia, así como el impacto de este uso en la crianza de los hijos. A partir de la revisión realizada, se observó que los estudios sobre el tema señalan la importancia de la mediación y el control de los padres sobre la exposición de niños y adolescentes a las redes sociales y los juegos electrónicos, lo que resulta en un factor protector para el desarrollo. Además, se observó que los padres y los niños pueden diferir con respecto a la percepción del control parental ejercido.

Palabras clave: redes sociales, videojuegos, estilos parentales, infancia, adolescencia.

As Tecnologias de Informação e Comunicação (TICs) têm sido consideradas componentes centrais na vida de crianças e de adolescentes (Ohannessian, 2009), modificando as formas de comunicação e de socialização (Ungerer, 2013). Evidências sugerem que a exposição às mídias pode influenciar comportamentos e atitudes, tanto de forma positiva quanto negativa (Padilla-Walker, Coyne, Fraser, Dyer, \& Yorgason, 2012).

Alguns riscos têm causado preocupação entre os responsáveis, como o tempo de uso excessivo, predominância dos relacionamentos virtuais em relação ao contato face a face e situações de violência online (Leung \& Lee, 2012; Williams \& Stelko-Pereira, 2013). Destaca-se que a utilização de estratégias parentais para monitorar e supervisionar o uso da internet pelos filhos pode auxiliar na redução de comportamentos de risco online. Entretanto, a eficácia de tais estratégias costuma variar de acordo com alguns fatores, como a idade do adolescente e a qualidade da relação entre pais e filhos (Livingstone \& Helsper, 2008). Além disso, o nível de conhecimento digital dos pais pode impactar no modo de orientação e 
supervisão (Wong, 2010). Dentro desse contexto, o objetivo principal deste artigo foi descrever e discutir o uso das redes sociais e da internet na infância e na adolescência, assim como o impacto deste uso na parentalidade.

\section{Uso de Tecnologias por Crianças e Adolescentes}

O desenvolvimento das novas tecnologias ocasionou diversas mudanças na sociedade, e, sobretudo, na geração de crianças e adolescentes nascidos em meio a essa nova realidade, que, inclusive, receberam definições sociológicas e publicitárias como "Nativos Digitais" e “Geração Z”, em razão de sua familiaridade com as tecnologias digitais (Kämpf, 2011). Tal geração é caracterizada por ter grande acesso às tecnologias, tendo sido exposta e estimulada desde a idade precoce ao uso de equipamentos tecnológicos (Spizzirri, Wagner, Mosmann, \& Armani, 2012).

A partir de um levantamento realizado pelo Nielsen Online (Ibope, 2010), concluiu-se que o número de internautas vem crescendo de forma acelerada no Brasil. De acordo com a pesquisa, em agosto de 2010 o número de usuários ativos era de 41,5 milhões, representando em torno de $22 \%$ da população. Também foi concluído que os internautas brasileiros passam mais de 60 horas por mês conectados à internet, sendo as redes sociais um dos sites mais utilizados. Com relação às crianças e aos adolescentes, a ONG SaferNet Brasil (2008), a partir de um levantamento realizado com 1.400 crianças, adolescentes e pais, aponta que $64 \%$ dos respondentes relatam utilizar a internet no próprio quarto, com total privacidade. Além disso, 87\% afirmam não possuir nenhum tipo de restrição quanto ao uso da internet.

Diversos benefícios e riscos têm sido associados ao uso da internet e de jogos eletrônicos. Entre os principais benefícios encontram-se a aquisição de línguas estrangeiras (García-Carbonel, Rising, Montero, \& Watts, 2001), melhora nas funções executivas (Kuhn et al., 2014), na atenção e nas habilidades viso-espaciais (Dye, Green, \& Bavelier, 2009), e aprimoramento nas habilidades de leitura (Jackson, Eye, Witt, Zhao, \& Fitzgerald, 2011). Por outro lado, são apontadas como possíveis consequências negativas o desenvolvimento de problemas físicos, sociais e psicológicos (Çankayaa \& Odaba, 2009), assim como agressividade (Anderson et al., 2010), dificuldade nas relações interpessoais (Padilla-Walker, Nelson, Carroll, \& Jensen, 2010) e distúrbios do sono (Ivarsson, Anderson, Akerstedt, \& Lindblad, 2013).

Resultados de um estudo (Spizzirri et al., 2012) que avaliou a percepção de adolescentes em relação a aspectos positivos no uso da internet, concluíram que o maior 
benefício apontado é a rapidez na comunicação. Outras vantagens, indicadas pelos adolescentes, fazem referência à internet ser uma tecnologia econômica e à possibilidade de conhecer pessoas novas, aumentando o número de amigos. Matos (2015) refere, por meio da compilação de falas de adolescentes portugueses, mais algumas vantagens, como a oportunidade de interação social, ainda que exista distância geográfica, e a possibilidade de relacionamentos afetivos iniciarem através das tecnologias.

Alguns dos possíveis riscos associados especificamente à comunicação online referem-se ao cyberbullying e a questões sexuais, como por exemplo, o recebimento de solicitações sexuais indesejadas ou o assédio (Valkenburg \& Peter, 2011). Além disso, há preocupação por parte dos adultos em relação à exposição dos adolescentes na internet, como o envio ou divulgação de fotos íntimas, e o acesso a conteúdos inadequados acerca de diversos temas, como transtornos alimentares ou uso de drogas (Lima, 2011).

O cyberbullying tem sido definido de forma semelhante ao fenômeno do bullying, conceituado por Olweus (1993). Dessa forma, o cyberbullying refere-se a comportamentos agressivos, intencionais e repetitivos, os quais são realizados por um grupo ou por um indivíduo contra uma vítima que possui dificuldade para se defender, caracterizando uma relação desigual de poder (Smith et al., 2008). Diferente do processo de bullying, que costuma ocorrer face a face, estes atos ocorrem através de meio eletrônicos, principalmente pela internet e telefones celulares (Slonje \& Smith, 2008).

É comum as agressões virtuais ocorrerem de forma anônima, por meio de perfis falsos nas redes sociais. Assim, o agressor não precisa ser mais forte fisicamente do que a vítima, o que ocorre com frequência em situações de bullying (Wanzinack, 2014). Outra diferença que se destaca entre o bullying e o cyberbullying é a questão da previsibilidade. No processo de bullying, que muitas vezes ocorre em ambiente escolar, é possível prever em quais situações o aluno pode se encontrar em risco, como por exemplo, momentos em que não há adultos por perto. Já através dos meios eletrônicos, geralmente não há possibilidade de prever ou tentar evitar tais situações (Smith, 2010). Atos de cyberbullying podem incluir o envio de mensagens para o celular ou para o e-mail da vítima, assim como a publicação de fotos e vídeos íntimos ou constrangedores nas redes sociais (Menesini et al., 2012). Em relação à exposição de fotos e de vídeos íntimos, sabe-se que estes podem tomar grande proporção no mundo virtual, podendo ser divulgados em diversas redes sociais, como o facebook, twitter, youtube, entre outras (Wanzinack, 2014).

Resultados da pesquisa TIC Kids Online Brasil (2012) mostraram que o uso da internet com o objetivo de realizar trabalhos escolares é uma prática esporádica, sendo que 
$49 \%$ dos respondentes fazem uso uma ou duas vezes por semana, e $38 \%$ uma ou duas vezes por mês, com essa finalidade. Por outro lado, $53 \%$ das crianças e dos adolescentes referiram utilizar as redes sociais e a troca de mensagens diariamente. Mais de um terço dos participantes também utiliza a internet para jogar e ouvir músicas.

Passarelli, Junqueira e Angeluci (2014) destacam algumas diferenças com relação às principais ações de crianças e de adolescentes do sexo feminino e masculino na internet. $\mathrm{O}$ uso de games e download de arquivos de música parece estar relacionado com atividades mais realizadas por meninos. Já as meninas, muitas vezes se envolvem mais com atividades referentes ao âmbito social, como falar ao celular, enviar mensagens e participar de chats. Apesar dessas diferenças, Gross (2004) concluiu, a partir de uma pesquisa realizada com 261 adolescentes, que não há diferenças entre os sexos acerca do tempo em que meninas e meninos passam navegando na internet. Portanto, os resultados indicam que os adolescentes de ambos os sexos permanecem online por uma quantidade de tempo semelhante.

Além das redes sociais, a utilização de videogame também vem crescendo de forma significativa (Coyne, Padilla-Walker, Stockdale \& Day, 2011), gerando preocupações acerca dos possíveis riscos, como os efeitos dos jogos com conteúdo violento e o longo tempo despendido nessa atividade (Grüsser, Thalemann \& Griffiths, 2007). Pesquisas realizadas acerca do tema, incluindo meta-análises e estudos longitudinais, sugerem que jogos com conteúdo violento possuem associação positiva com agressividade e associação negativa com o rendimento acadêmico (Anderson, 2004; Moller, \& Krahe, 2009).

As tecnologias têm se mostrado um novo desafio tanto para os filhos quanto para os pais (Verzoni \& Lisboa, 2017), que possuem papel fundamental ao auxiliá-los e orientá-los. O uso da internet está relacionado às práticas e aos estilos parentais, de modo que o fornecimento de orientações, assim como a comunicação positiva entre pais e filhos, apresenta associações com menor probabilidade da ocorrência de comportamentos de risco na internet (Smith, Gradisar, \& King, 2015). Niquice (2014) ressalta que orientações por parte dos responsáveis sobre os riscos que as crianças e os adolescentes correm são extremamente necessárias.

\section{Parentalidade em Tempos de Redes Sociais e de Internet}

Diversos modelos têm sido utilizados para investigar e desenvolver a forma como os pais lidam com os filhos. As práticas educativas são as estratégias utilizadas pelos pais para lidarem diretamente com o comportamento dos filhos (Holden, 2010). Os primeiros trabalhos 
que definiram as práticas educativas parentais a partir das categorias indutivas e coercitivas foram realizados por Hoffman, na década de 70 (Hoffman, 1975). De acordo com o autor, a partir das práticas indutivas os responsáveis dialogam com os filhos sobre as consequências dos comportamentos, possibilitando uma reflexão. A utilização de práticas indutivas é vista de forma positiva para o desenvolvimento das crianças, gerando senso de respeito diante de opiniões e de perspectivas distintas (Mondin, 2008).

As práticas coercitivas envolvem a disciplina relacionada à punição física, força e privações de afeto e de privilégios (Hoffman, 1975). Tais práticas costumam gerar emoções desagradáveis nas crianças e nos adolescentes, como medo, raiva e ansiedade, podendo dificultar que compreendam a situação e a importância de ocorrerem mudanças no comportamento.

Gomide (2006) faz referência às práticas parentais positivas e negativas. Nesse modelo, as práticas educativas positivas incluem a monitoria positiva, que se relaciona ao uso adequado de atenção aos filhos e ao fornecimento de privilégios, e também ao comportamento moral, que envolve propiciar condições favoráveis ao desenvolvimento de virtudes, como a empatia, a responsabilidade e a justiça. A imposição de regras e de limites na relação familiar está associada a aspectos como clareza, coerência e supervisão. As práticas educativas negativas estão associadas à negligência, falta de atenção e de expressão de afeto, disciplina inconsistente, assim como práticas corporais negativas (Gomide, 2006), podendo gerar efeitos nocivos, como emoções de raiva e medo, além de comportamentos de esquiva.

Os estilos parentais constituem-se em outro modelo teórico utilizado para investigar as relações entre os pais e os filhos. Os estudos acerca dos estilos parentais iniciaram com os trabalhos de Baumrind (1966) e se consolidaram com a proposta de Maccoby e Martin (1983). O estilo parental pode ser definido como um conjunto de atitudes dos pais direcionadas aos filhos, as quais criam um clima emocional nos quais os comportamentos dos pais são expressos (Darling \& Steinberg, 1993).

De acordo com Maccoby e Martin (1983), um estilo parental envolve diversas práticas, além de aspectos como a comunicação não verbal e frequência de expressões de afeto. Tais autores destacam que esses estilos variam entre duas dimensões: responsividade e exigência. A responsividade se caracteriza por comportamentos de expressão de afeto, envolvimento e conhecimento acerca das atividades dos filhos, além da utilização de reforço positivo. Já a exigência pode ser caracterizada por práticas de supervisão, monitoramento e disciplina (Baumrind, 1966). A combinação das dimensões responsividade e exigência 
derivaram quatro estilos parentais: autoritário, autoritativo, negligente e indulgente (Darling \& Steinberg, 1993).

O estilo parental autoritário envolve comportamentos intrusivos, o uso de punição e grau elevado grau de exigência. No estilo parental indulgente, percebe-se baixa exigência, alta responsividade e expressão de afeto, e estabelecimento de limites dado de maneira inconsistente (Yang, Chankon, Laroche, \& Lee, 2014). Por sua vez, o estilo parental negligente se caracteriza por comportamentos de baixa responsividade e de pouca demonstração de afeto (Yang et al., 2014). Por fim, os pais com estilo autoritativo conseguem conciliar as dimensões de responsividade e exigência de forma saudável. Sendo assim, há demonstração de afeto, definição de limites e regras de maneira clara e utilização de reforço positivo. Este estilo parental se caracteriza pela comunicação aberta entre pais e filhos. Os pais demonstram aceitação da gradual independência de seus filhos, de forma que são exigentes, mas ao mesmo tempo, responsivos (Becoña et al., 2012).

O processo de educar filhos envolve lidar com diferentes comportamentos que se modificam ao longo do crescimento e, ao fazerem isso, os pais estão empregando as práticas educativas que conhecem e exercendo um estilo parental. A forma como os pais lidam com os comportamentos dos filhos pode se constituir em um fator de risco ou de proteção ao desenvolvimento. Dessa forma, a questão que se coloca é como os pais podem gerenciar o comportamento dos filhos ao usarem internet, redes sociais ou jogos eletrônicos de modo a promover um desenvolvimento saudável? Percebe-se que a utilização crescente da internet por crianças e adolescentes tem imposto desafios relacionados à parentalidade. Nos dias atuais, com frequência pais e filhos se envolvem em conflitos, visto que os responsáveis se preocupam com os efeitos negativos da internet sobre a segurança e os comportamentos das crianças (Livingstone \& Helsper, 2008).

A mediação parental no uso da internet pode ser definida como as estratégias que os responsáveis utilizam com a finalidade de aumentar os benefícios e diminuir os riscos das crianças e dos adolescentes frente às tecnologias (Kirwil, 2009). Considera-se ampla a variabilidade entre as formas de supervisão e de monitoramento parentais em relação às tecnologias, podendo variar entre métodos como o uso de filtros para determinados sites, definição de horários de uso, até a proibição do uso desacompanhada de um adulto (Spizzirri et al., 2012).

Maidel e Vieira (2015) empregam o termo mediação ao se referirem a condutas e práticas realizadas pelos pais que influenciam a forma como os filhos pensam a respeito das mídias. Três tipos de mediação foram elaborados por Valkenburg, Krcmar, Peeters e 
Marseille (1999), sendo nomeadas de Mediação Ativa (MA), Mediação Restritiva (MR) e Mediação "Uso Acompanhado" (UA). Na mediação ativa há diálogo entre pais e filhos, assim como o fornecimento de orientações e discussões sobre as atividades online e os conteúdos acessados. Este tipo de mediação possui como objetivo discutir acerca dos conteúdos disponíveis na internet, auxiliando as crianças e os adolescentes a desenvolverem um pensamento crítico sobre o material acessado (Collier et al., 2016).

Por outro lado, a mediação restritiva se refere ao estabelecimento de regras acerca do uso da internet, podendo incluir restrições acerca do tempo despendido, local de uso e conteúdo acessado. Nesse tipo de mediação nem sempre há discussão e diálogo com os filhos acerca das regras estabelecidas. Já na mediação "uso acompanhado", um dos responsáveis permanece presente e acompanha a criança durante a atividade, porém, sem dialogar sobre os conteúdos acessados (Livingstone \& Helsper, 2008). Os pais podem utilizar uma combinação desses tipos de mediações, por exemplo, discutindo os conteúdos e o tipo de comportamento cuidadoso que a criança pode ter no uso da internet, ao mesmo tempo em que restringe o acesso a horários e dias específicos. Maidel e Vieira (2015) denominaram de 'mediação mista' essa combinação. A mediação "uso acompanhado" pode ser utilizada com mais frequência com crianças menores.

A partir da meta-análise realizada por Collier et al. (2016), encontrou-se que a mediação restritiva desempenha um papel pequeno, mas ainda assim estatisticamente significativo na prevenção de resultados negativos advindos do uso da internet. A significância se mostrou maior em relação ao tempo despendido nas tecnologias e a comportamentos sexuais de risco online. Além disso, a mediação restritiva se mostrou efetiva para diminuir o tempo conectado, e também para reduzir a exposição a conteúdos inapropriados para a idade, como conteúdos sexuais. De forma semelhante, a mediação ativa pode ser considerada como um fator protetor frente aos efeitos negativos das tecnologias relacionados à agressividade e a comportamentos sexuais. No entanto, em relação ao tempo utilizado em atividades online, a mediação ativa não apresentou influência significativa (Collier et al., 2016). Possivelmente, porque na mediação ativa, os pais foquem no diálogo e na concessão de informações acerca de aspectos envolvidos na exposição à internet, objetivando promover autorregulação e responsabilidade nos filhos.

A mediação parental das mídias parece ter mais efeitos positivos quando os pais se envolvem de forma ativa, participando da escolha dos sites e acompanhando os filhos durante as atividades. Apenas restringir ou proibir não tem mostrado tanta eficácia no processo de mediação e orientação das crianças e dos adolescentes em relação ao uso da internet (Lee \& 
Chae, 2007). Além disso, a mediação e limites fornecidos pelos pais não deve focar apenas na diminuição do tempo de exposição à internet, mas também na ampliação de outras atividades importantes para o desenvolvimento, como a prática de esporte, a leitura de revistas e de livros (Len-Ríos, Hughes, McKee, \& Young, 2015).

Desiderá e Zuben (2013) destacam outras estratégias que podem ser utilizadas pelos responsáveis, como por exemplo, manter o computador em um local público da casa, a fim de que seja possível observar mais de perto, assim como orientar os filhos e participar de algumas atividades. Além disso, há a possibilidade de ativação da função "controle para pais", a qual está presente em alguns sistemas. Esse mecanismo pode evitar que as crianças e os adolescentes acessem conteúdos inapropriados para a faixa etária. Fundamentalmente, os pais precisam se interessar em conhecer o conteúdo acessado pelos filhos, o nível de exposição que as ferramentas digitais proporcionam durante o uso e os recursos que dispõem, a fim de utilizá-los para auxiliar no controle e na proteção.

Dishion, Nelson e Bullock (2004) distinguem as estratégias de restrição e de monitoramento parental em relação aos possíveis riscos na internet. Enquanto a restrição se refere apenas a limitar o tempo despendido online, o monitoramento diz respeito a maiores esforços dos pais para proteger os filhos de perigos no mundo online. Tais práticas de monitoramento incluem tanto a restrição do acesso à internet quanto à delimitação de regras sobre o uso, como tempo e conteúdos acessados (Khurana, Bleakley, Jordan, \& Romer, 2015).

Em relação ao contexto físico e social do cotidiano de crianças, resultados do estudo de Maidel e Vieira (2015) mostraram um favorecimento por parte dos responsáveis para que as crianças tenham acesso às tecnologias, tanto por haver muitos aparelhos eletrônicos em casa quanto por falta de controle parental. Além disso, considera-se mais difícil tornar o uso da internet e de redes sociais uma atividade compartilhada, em comparação com o uso da televisão e de videogame. Tal dificuldade pode ocorrer devido a diferentes fatores, como o tamanho da tela, a posição em que se senta para realizar a atividade, e à possibilidade de abrir diferentes janelas na tela do computador, aspecto que pode dificultar o monitoramento (Livingstone \& Helsper, 2008).

De acordo com as percepções das próprias crianças e dos adolescentes participantes da pesquisa TIC Kids Online Brasil 2012 (2013), 38\% acreditam que os pais sabem muito bem o que eles fazem na internet; $35 \%$ pensam que os pais sabem mais ou menos; $17 \%$ percebem que os pais sabem apenas um pouco; 10\%, que os pais não sabem. Já em relação ao que as crianças percebem sobre o tipo de orientação fornecida pelos pais, 74\% da amostra afirmou 
que os pais conversam a respeito do uso da internet; $54 \%$ referem que os pais se mantêm por perto durante as atividades online; $45 \%$ sentam junto com os filhos (somente observando o que está sendo realizado); 35\% estimulam o uso da internet e o autodidatismo em relação a ela; e $35 \%$ realizam atividades juntamente com os filhos na internet.

Ainda sobre o monitoramento parental, Tabone e Messina (2010) pesquisaram crianças com idade entre dez e onze anos, e encontraram que estas não percebem a participação dos pais em relação às suas atividades online, a justificativa para isso pode estar relacionada à percepção de autonomia das crianças, a confiança dos pais nos filhos em relação às mídias, ou a falta de limites e de supervisão parentais. Outra hipótese para a menor mediação parental refere-se ao número de filhos. Estudos sugerem que, em famílias maiores, os pais exercem menos controle e supervisão com relação ao uso da internet (Alqahtani, Atkinson, Furnell, \& Stengel 2017).

Em um estudo realizado com adolescentes e seus pais, Liau, Khoo e Ang (2008) encontraram que os pais costumam subestimar o engajamento dos adolescentes em comportamentos de risco, como marcar encontros com conhecidos virtuais, ou visitar sites inapropriados, e superestimar o monitoramento da internet realizado por eles com os filhos adolescentes. Alguns pais consideram que estão supervisionando o uso da internet de forma apropriada, quando estão apenas realizando ações de checagem, como verificar o que há na tela do dispositivo no momento em que o jovem o está utilizando. Para os autores, a ideia de monitoramento precisa ser redefinida e clarificada para os pais, os incentivando a desenvolver habilidades de comunicação com os seus filhos sobre o uso da internet (Liau et al., 2008).

Tais ideias advindas de alguns pais, de que os filhos têm capacidade para se autogerir na internet, podem estar associadas à idade das crianças e dos adolescentes. Estudos indicam que crianças com menos de onze anos costumam ser mais supervisionadas e monitoradas de perto pelos responsáveis (John, 1999), além de aceitarem mais facilmente as restrições impostas (Nathanson, 2001). A mediação parental tende a diminuir à medida que as crianças crescem, de modo que geralmente são empregadas menos estratégias de mediação na adolescência em comparação à infância (Bocking \& Bocking, 2009), o que, de certa forma, é esperado dado que, na adolescência, espera-se maior capacidade de autorregulação e de senso de crítico quanto aos riscos possíveis.

No entanto, a falta de monitoramento parental é considerada um fator de risco significativo para a ocorrência de situações como a perpetração e a vitimização online (Korchmaros, Mitchell, \& Ybarra, 2014). Esse dado foi corroborado pelos estudos de Khurana et al. (2015), cujos resultados apontaram uma associação entre práticas parentais de 
monitoramento e estabelecimento de regras acerca do uso da internet com menor probabilidade de riscos para os adolescentes, como o assédio online. Considera-se que os pais previnem de modo mais efetivo os riscos online se estiverem envolvidos e informados acerca da vida de seus filhos, do que se apenas restringirem comportamentos na internet. Deve-se considerar também que, embora a influência parental possa diminuir os riscos online, as crianças e os adolescentes são afetados por outros agentes como amigos e a mídia (Wang, Iannotti, \& Nansel, 2009).

Cardoso, Espanha e Lapa (2008) indicam outras questões referentes ao uso da internet que comumente são razões de conflito entre pais e filhos, como o tempo despendido nas mídias. Considerando-se tal panorama, destaca-se a importância da regulação parental relacionada ao uso saudável das tecnologias. Nesse sentido, em relação aos jogos eletrônicos, é importante que os pais atentem para possíveis excessos no uso de jogos, compreendam como se dá a interação dos filhos com outros jogadores (no caso dos jogos online) e pensem juntamente com as crianças e os adolescentes na escolha destes jogos. Para isso, considera-se essencial o estabelecimento de relações positivas entre pais e filhos, incluindo momentos de trocas e de diálogos abertos (Lima, 2011). De modo semelhante aos jogos, é importante que os pais possam conhecer os sites e as redes sociais das quais seus filhos desejam participar, a fim de verificar se são adequados para suas respectivas idades (O'Keeffe, Clarke-Pearson, \& Council on Communications and Media, 2011).

\section{Considerações Finais}

Essa revisão bibliográfica teve como o objetivo principal descrever e discutir o uso das redes sociais e jogos eletrônicos na infância e na adolescência, assim como o impacto deste uso na parentalidade. O papel dos pais, ao gerir a utilização das mídias pelas crianças e pelos adolescentes, é considerado fundamental, pois esses são os principais organizadores da vida dos filhos e o domicílio é o local em que os jovens mais utilizam a internet (Tabone \& Messina, 2010). Dessa forma, além dos pais oportunizarem o acesso às tecnologias aos filhos, também costumam ser os responsáveis em administrar tal uso, o que inclui a definição de normas, orientações, supervisão e monitoramento (Maidel \& Vieira, 2015).

Liau, Khoo e Hwaang (2005) concluíram que o fornecimento de regras por parte dos pais em relação ao uso da internet, como orientações aos filhos sobre não se comunicar com pessoas desconhecidas, está relacionado com a diminuição da probabilidade da ocorrência de comportamentos de risco na internet, como por exemplo, marcar encontros face a face com 
pessoas com as quais se relacionam no mundo virtual. Do mesmo modo, tais autores sugerem que relações positivas e de confiança nas quais os filhos relatam aos pais quando recebem mensagens inapropriadas ou convites de amizade de pessoas desconhecidas, também pode contribuir para a diminuição de tais comportamentos.

Embora exista mediação parental em relação ao uso da internet, nem sempre há eficácia, visto que alguns pais tendem a restringir determinadas atividades apenas quando o filho se envolve em algum problema. Portanto, considera-se importante refletir sobre as estratégias utilizadas a fim de que estas sejam benéficas e protetoras, e não intrusivas (Livingstone, 2007; Livingstone \& Helsper, 2008). A partir desta revisão, percebe-se que o monitoramento parental pode representar um desafio para os pais, constituindo-se em um repertório a ser aprendido. Além disso, a mediação ativa combinada com a mediação restritiva e o monitoramento parece ter um efeito protetiva para o uso saudável das tecnologias. Nesse tipo de mediação os responsáveis se fazem presentes e participam ativamente com os filhos das escolhas das tecnologias utilizadas, delimitando regras acerca do uso sempre que necessário.

Verzoni e Lisboa (2017) sugerem que sejam realizadas intervenções com os pais, como o fornecimento de orientações e de psicoeducação acerca dos riscos e dos benefícios acerca do uso das tecnologias. Dessa forma, os responsáveis podem lidar de forma mais segura com os filhos, participando de sua vida virtual, porém, libertando-se de "préconceitos" e estando atentos para não agir de forma invasiva. Inicialmente, antes de intervir de forma impositiva e restritiva acerca do uso das tecnologias, é importante agir de modo preventivo (Wendt \& Lisboa, 2013). A partir do momento, em que os responsáveis forem informados e tiverem conhecimento acerca dos efeitos positivos e negativos advindos das tecnologias, poderão pensar na criação de regras apropriadas para o uso da internet, discutir a escolha dos jogos e sites, e também participar, realizando algumas atividades online junto dos filhos (Collier et al., 2016).

Portanto, o uso das tecnologias pode ser considerado positivo, oferecendo experiências enriquecedoras e sendo um facilitador para a aprendizagem, assim como pode envolver riscos devido à exposição das crianças e dos adolescentes (Spizzirri, et al., 2012). Percebidos tais riscos, considera-se essencial a participação e o monitoramento parental a fim de auxiliar seus filhos a fazerem uso das tecnologias de forma saudável e positiva. A partir de tais achados, reforça-se a importância da realização de práticas de conscientização e de esclarecimento para pais, profissionais, professores, e para as próprias crianças e adolescentes acerca do uso das tecnologias. 


\section{Referências}

Alqahtani, N., Atkinson, S., Furnell, S., \& Stengel, I. (2017). Internet Risks for Children: Parents' Perceptions and Attitudes: An Investigative Study of the Saudi Context. Internet Technologies and Applications (ITA), 98-103. doi: 10.1109/ITECHA.2017.8101918

Anderson, C. (2004). An update on the effects of playing violent video games. Journal of Adolescence, 27(1), 113-122. doi: 10.1016/j.adolescence.2003.10.009

Anderson, C., Berkowitz, L., Donnerstein, E., Huesmann, L., Johnson, J., Linz, D., \& Wartella, E. (2003). The influence of media violence on youth. Psychological Science in the Public Interest, 4(3), 81-110. doi: 10.1111/j.1529-1006.2003.pspi_1433.x

Anderson, C., Shibuya, A., Ihori, N., Swing, E., Bushman, B., Sakamoto, A., ... Saleem, M. (2010). Violent videogame effects on aggression, empathy, and prosocial behavior in eastern and western countries: A meta-analytic review. Psychological Bulletin, 136(2), 151-173. doi: 10.1037/a0018251

Baumrind, D. (1966). Effects of authoritative parental control on child behavior. Child Development, 37(4), 887-907. doi: 10.2307/1126611

Becoña, E., Martínez, Ú., Calafat, A., Juan, M., Fernández-Hermida, J., \& Secades-Villa, R. (2012). Parental styles and drug use: A review. Drugs: Education, prevention and policy, 19(1), 1-10. doi: 10.3109/09687637.2011.631060

Bocking, S., \& Bocking, T. (2009). Parental mediation of television: Test of a Germanspeaking scale and finding on the impact of parental attitudes, sociodemographic and family factors in German-speaking Switzerland. Journal of Children and Media, 3(3), 286-302. doi: 10.1080/17482790902999959

Çankaya, S., \& Odaba, H. (2009). Parental controls on children's computer and Internet use. Procedia - Social and Behavioral Sciences, 1(1), 1105-1109. doi: 10.1016/j.sbspro.2009.01.199

Cardoso, G., Espanha, R., \& Lapa, T. (2008). Dinâmica familiar e interacção em torno dos media: autonomia dos jovens, autoridade e controlo parental sobre os media em Portugal. Comunicação e Sociedade, 13, 31-53. doi:10.17231/comsoc.13(2008).1143

Collier, K., Coyne, S., Rasmussen, E., Hawkins, A., Padilla-Walker, L., Erickson, S., \& Memmott-Elison, M. (2016). Does parental mediation of media influence child outcomes? A meta-analysis on media time, aggression, substance use, and sexual behavior. Developmental psychology, 52(5), 798-812. doi: 10.1037/dev0000108 
Coyne, S., Padilla-Walker, L., Stockdale, L., \& Day, R. (2011). Game On...Girls: Associations Between Co-playing Video Games and Adolescent Behavioral and Family Outcomes. Journal of Adolescent Health, 49, 160-165. doi: 10.1016/j.jadohealth.2010.11.249

Darling, N., \& Steinberg, L. (1993). Parenting style as context: An integrative model. Psychological Bulletin, 113(3), 487-496. doi: 10.1037/0033-2909.113.3.487

Desiderá, L., \& Zuben, M. (2013). Crianças e adolescentes: Usando a internet com segurança. In A. Barbosa (Coord.), TIC Kids Online Brasil 2012: Pesquisa sobre o uso da internet por crianças e adolescentes (pp. 65-72). São Paulo: Comitê Gestor da Internet no Brasil.

Dishion, T., Nelson, S., \& Bullock, B. (2004). Premature adolescent autonomy: Parent disengagement and deviant peer process in the amplification of problem behaviour. Journal of Adolescence, 27(5), 515-530. doi: 10.1016/j.adolescence.2004.06.005

Dye, M., Green, C., \& Bavelier, D. (2009). The development of attention skills in action video game players. Neuropsychologia, 47(8-9), 1780-1789. doi: 10.1016/j.neuropsychologia.2009.02.002

García-Carbonell, A., Rising, B., Montero, B, \& Watts, F. (2001). Simulation/gaming and the acquisition of communicative competence in another language. Simulation \& Gaming, 32(4), 481-491. doi: 10.1177/104687810103200405

Gomide, P. (2006). Inventário de Estilos Parentais: Modelo teórico: Manual de aplicação, apuração e interpretação. Petrópolis, RJ: Vozes.

Gross, E. (2004). Adolescent Internet use: What we expect, what teens report. Journal of Applied Developmental Psychology, 25(6), 633-649. doi: 10.1016/j.appdev.2004.09.005

Grusser, S., Thalemann, R., \& Griffiths, M. (2007). Excessive computer game playing: Evidence for addiction and aggression? Cyber Psychology \& Behavior, 10(2), 290292. doi: $10.1089 /$ cpb.2006.9956

Hoffman, M. (1975). Moral internalization, parental power, and the nature of parent-child interaction. Developmental Psychology, 11(2), 228-239. doi: 10.1037/h0076463

Holden, G. (2010). Parenting: A dynamic perspective. Thousand Oaks: Sage.

IBOPE (2010). Pesquisa Ibope Nielsen Online. São Paulo: IBOPE. Recuperado de http://www.ibope.com.br/calandraWeb/servlet/CalandraRedirect?temp=5\&proj=Portal IBOPE $\&$ pub $=\mathrm{T} \& \mathrm{db}=\mathrm{caldb} \&$ Comp $=$ Not\%EDcias $\&$ docid $=0$ A276B95D145374B 83257 7B6007A5F6A 
Ivarsson, M., Anderson, M., Akerstedt, T., \& Lindblad, F. (2013). The effect of violent and nonviolent video games on heart rate variability, sleep, and emotions in adolescents with different violent gaming habits. Psychosomatic Medicine, 75(4), 390-396. doi: 10.1097/PSY.0b013e3182906a4c

Jackson, L., Eye, A., Witt, E., Zhao, Y., \& Fitzgerald, H. (2011). A longitudinal study of the effects of Internet use and videogame playing on academic performance and the roles of gender, race and income in these relationships. Computers in Human Behavior, 27(1), 228-239. doi: 10.1016/j.chb.2010.08.001

John, R. (1999). Consumer Socialization of Children: A Retrospective Look at Twenty-Five Years of Research. Journal of Consumer Research, 26(3), 183-213. doi: $10.1086 / 209559$

Kämpf, C. (2011). A geração Z e o papel das tecnologias digitais na construção do pensamento. ComCiência, (131), 10-12. Recuperado em http://comciencia.scielo.br/scielo.php?script=sci_arttext\&pid=S1519$76542011000700004 \& \operatorname{lng}=\mathrm{en} \& \mathrm{nrm}=\mathrm{iso}$

Khurana, A., Bleakley, A., Jordan, A., \& Romer, D. (2015). The Protective Effects of Parental Monitoring and Internet Restriction on Adolescents' Risk of Online Harassment. Journal of Youth and Adolescence, 44(5), 1039-1047. doi: 10.1007/s10964-014-02424

Kirwil, L. (2009). Parental Mediation of Children's Internet Use in Different European Countries. Journal of Children and Media, 3(4), 394-409. doi: $10.1080 / 17482790903233440$

Korchmaros, J., Mitchell, K., \& Ybarra, M. (2014). Technology-based interpersonal victimization: Predictors of patterns of victimization over time. Journal of Interpersonal Violence, 29(7), 1297-1317. doi: 10.1177/0886260513506277

Kühn, S., Lorenz, R., Banaschewski, T., Barker, G., Büchel, C., Conrod, P., ... Gallinat, J. (2014). Positive association of video game playing with left frontal cortical thickness in adolescents. PloS One, 9(3), e91506. doi: 10.1371/journal.pone.0091506

Lee, S., \& Chae, Y. (2007). Children's Internet use in a family context: Influence on family relationships and parental mediation. CyberPsychology \& Behavior, 10(5), 640-644. doi: $10.1089 / \mathrm{cpb} .2007 .9975$

Len-Ríos, M., Hughes, H., McKee, L., \& Young, H. (2016). Early adolescents as publics: A national survey of teens with social media accounts, their media use preferences, 
parental mediation, and perceived Internet literacy. Public Relations Review, 42(1), 101-108. doi: 10.1016/j.pubrev.2015.10.003

Leung, L., \& Lee, P. (2012). The influences of information literacy, internet addiction and parenting styles on internet risks. New Media \& Society, 14(1), 117-136. doi: $10.1177 / 1461444811410406$

Liau, A., Khoo, A., \& Hwaang, P. (2005). Factors Influencing Adolescents Engagement in Risky Internet Behavior. Cyber Psychology \& Behavior, 8(6), 513-520. doi: $10.1089 / \mathrm{cpb} .2005 .8 .513$

Liau, A., Khoo, A., \& Ang, P. (2008). Parental awareness and monitoring of adolescent Internet use. Current Psychology, 27(4), 217-233. doi: 10.1007/s12144-008-9038-6

Lima, A. (2011). Principais características dos comportamentos de risco online. In A. Lima, Cyberbullying e outros riscos na internet: Despertando a atenção de pais $e$ professores (pp. 121-127). Rio de Janeiro: Wak.

Livingstone, S. (2007). Strategies of parental regulation in the media-rich home. Computers in Human Behavior, 23(3), 920-941. doi: 10.1016/j.chb.2005.08.002

Livingstone, S., \& Helsper, E. (2008). Parental mediation of children's internet use. Journal of Broadcasting \& Electronic Media, 52(4), 581-599. doi: $10.1080 / 08838150802437396$

Maccoby, E., \& Martin, J. (1983). Socialization in the context of the family: Parent-child interaction. In E. M. Hetherington (Org.), Handbook of child psychology (Vol. 4, pp. 1-110). New York: Wiley.

Maidel, S., \& Vieira, M. (2015). Mediação parental do uso da internet pelas crianças. Psicologia em Revista, 21(2), 293-313. doi: 10.5752/P.1678-9523.2015V21N2P292

Matos, M. (2015). Adolescentes em navegação segura, por águas desconhecidas. Lisboa: Coisas de Ler.

Menesini, E., Nocentini, A., Palladino, B., Frisén, A., Berne, S., Ortega-Ruiz, R., ... Smith, P. (2012). Cyberbullying definition among adolescents: A comparison across six European countries. Cyberpsychology, Behavior, and Social Networking, 15(9), 455463. doi: 10.1089/cyber.2012.0040

Moller, I., \& Krahé, B. (2009). Exposure to violent games and aggression in german adolescents: A longitudinal analysis. Aggressive Behavior, 35(1), 75-89. doi: 10.1002/ab.20290

Mondin, E. (2008). Práticas educativas parentais e seus efeitos na criação dos filhos. Psicologia Argumento, 26(54), 233-244. Recuperado de 
https://periodicos.pucpr.br/index.php/psicologiaargumento/article/viewFile/19885/191 87

Nathanson, A. (2001). Parents versus peers exploring the significance of peer mediation of antisocial television. Communication Research, 28(3), 25-274 doi: $10.1177 / 009365001028003001$

Niquice, F. (2014). Comportamentos de risco na adolescência. In L. F. Habigzang, E. Diniz, \& S. Koller (Orgs.), Trabalhando com adolescentes: Teoria e Intervenção psicológica (pp. 42-53). Porto Alegre: Artmed.

Ohannessian, C. (2009). Media use and adolescent psychological adjustment: An examination of gender differences. Journal of child and family studies, 18(5), 582-593. doi: $10.1007 / \mathrm{s} 10826-009-9261-2$

O’Keeffe, G., Clarke-Pearson, K., \& Council on Communications and Media. (2011). Clinical Report- The impact of Social Media on Children, Adolescents, and Families. American Academy of Pediatrics, 127(4), 800-804. doi: 10.1542/peds.2011-0054

Olweus, D. (1993). Bullying at school: What we know and what we can do. Oxford, Cambridge, MA: Blackwell.

Padilla-Walker, L., Coyne, S., Fraser, A., Dyer, W., \& Yorgason, J. (2012). Parents and adolescents growing up in the digital age: Latent growth curve analysis of proactive media monitoring. Journal of adolescence, 35(5), 1153-1165. doi: 10.1016/j.adolescence.2012.03.005

Padilla-Walker, L., Nelson, L., Carroll, J., \& Jensen, A. (2010). More Than a Just a Game: Video Game and Internet Use During Emerging Adulthood. Journal of Youth \& Adolescence, 39(2), 103-113. doi: 10.1016/j.adolescence.2012.03.005

Passarelli, B., Junqueira, A., \& Angeluci, A. (2014). Os nativos digitais no Brasil e seus comportamentos diante das telas. Matrizes, 8(1), 159-178. doi: 10.11606/issn.19828160.v8i1p159-178

SaferNet Brasil. (2008). RELEASE: 87\% dos jovens afirmam que não possuem restrições para uso da Internet. Recuperado de http://www.safernet.org.br/twiki/bin/view/SaferNet/Noticia20081009202936

Slonje, R., \& Smith, P. (2008). Cyberbullying: And-other main type of bullying? Scandinavian Journal of Psychology, 49(2), 147-154. doi: 10.1111/j.14679450.2007.00611.x

Smith, L., Gradisar, M., \& King, D. (2015). Parental Influences on Adolescent Video Game Play: A Study of Accessibility, Rules, Limit Setting, Monitoring, and Cybersafety. 
Cyberpsychology, Behavior, and Social Networking, 18(5), 273-279. doi: 10.1089/cyber.2014.0611

Smith, P. (2010). Cyberbullying: The European perspective. In J. Mora-Merchan \& T. Jaeger (Eds.), Cyberbullying: A cross-national comparison (pp. 7-19). Landau, Germany: Verlag Emprische Padagogik.

Smith, P., Mahdavi, J., Carvalho, M., Fisher, S., Russel, S., \& Tippett, N. (2008). Cyberbullying: Its nature and impact in secondary school pupils. Child Psychology and Psychiatry, 49(4), 376-385. doi: 10.1111/j.1469-7610.2007.01846.x

Spizzirri, R., Wagner, A., Mosmann, C., \& Armani, A. (2012). Adolescência conectada: Mapeando o uso da internet em jovens internautas. Psicologia Argumento, 30(69), 327-335.

https://periodicos.pucpr.br/index.php/psicologiaargumento/article/view/23288/22361

Tabone, S., \& Messina, L. (2010). Personal uses of internet and perceptions of parental mediation: A research with children 10 and 11 years old. Children, 2(2), 2077-2082. doi: 10.1016/j.sbspro.2010.03.284

TIC Kids Online Brasil 2012. (2013) Pesquisa sobre o uso da internet por crianças e adolescentes no Brasil. São Paulo: Cetic Brasil. Recuperado de https://cetic.br/media/docs/publicacoes/2/tic-kids-online-2012.pdf

Ungerer, R. (2013). Sociedade globalizada e mídia digital. In C. Abreu, E. Eisenstein, \& S. Estefenon (Orgs.), Vivendo esse Mundo Digital: Impactos na saúde, na educação e nos comportamentos sociais (pp. 21-30). Porto Alegre: Artmed.

Valkenburg, P., Krcmar, M., Peeters, A., \& Marseille, N. (1999). Developing a scale to assess three styles of television mediation: "Instructive mediation", "restrictive mediation", and "social coviewing". Journal of Broadcasting \& Electronic Media, 43(1), 52-66. doi: $10.1080 / 08838159909364474$

Valkenburg, P., \& Peter, J. (2011). Online Communication Among Adolescents: An Integrated Model of Its Attraction, Opportunities, and Risks. Journal of Adolescent Health, 48(2), 121-127. doi: 10.1016/j.jadohealth.2010.08.020

Verzoni, A., \& Lisboa, C. (2017). Digitalidade e tecnologia. In C. Neufeld (Org.), Terapia Cognitivo-Comportamental para Adolescentes: Uma perspectiva Transdiagnóstica e Desenvolvimental (pp. 286-298). Porto Alegre: Artmed.

Wang, J., Iannotti, R., \& Nansel, T. (2009). School bullying among adolescents in the united states: Physical, verbal, relational, and cyber. Journal of Adolescent Health, 45(4), 368-375. doi: 10.1016/j.jadohealth.2009.03.021 
Wanzinack, C. (2014). Bullying e cyberbullying: Faces silenciosas da violência. In Sierra J., \& Signorelli, M. (Orgs.), Diversidade e educação: Intersecções entre corpo, gênero e sexualidade, raça e etnia (pp. 67-81). Matinhos: UFPR Litoral.

Wendt, G., \& Lisboa, C. (2013). Agressão entre pares no espaço virtual: Definições, impactos e desafios do cyberbullying. Psicologia clínica, 25(1), 73-87. doi: 10.1590/S010356652013000100005

Williams, L., \& Stelko-Pereira, A. (2013). Por fora bela viola: Pesquisa e intervenção sobre cyberbullying. In C. Abreu, E. Eisenstein, \& S. Estefenon (Orgs.), Vivendo esse Mundo Digital: Impactos na saúde, na educação e nos comportamentos sociais (pp. 49-59). Porto Alegre: Artmed.

Wong, Y. (2010). Cyber-parenting: Internet benefits, risks and parenting issues. Journal of Technology in Human Services, 28(4), 252-273. doi: 10.1080/15228835.2011.562629

Yang, Z., Chankon, K., Laroche, M., \& Lee, H. (2014). Parental style and consumer socialization among adolescents: A cross-cultural investigation. Journal of Business Research, 67(1), 228-236. doi: 10.1016/j.jbusres.2013.05.008

\section{Endereço para correspondência}

\section{Fernanda Tabasnik Schwartz}

Rua Sarmento Leite, 245, Centro Histórico, Porto Alegre - RS, Brasil. CEP 90050-170

Endereço eletrônico: fernandatschwartz@ gmail.com

\section{Janaína Thais Barbosa Pacheco}

Rua Sarmento Leite, 245, Centro Histórico, Porto Alegre - RS, Brasil. CEP 90050-170

Endereço eletrônico: janaina.thais.pacheco@gmail.com

Recebido em: 06/04/2019

Reformulado em: 27/04/2020

Aceito em: 24/07/2020

\section{Notas}

* Mestrado em Psicologia e Saúde (UFCSPA).

** Professora adjunta/ Chefe do Departamento de Psicologia (UFCSPA).

Este artigo de revista Estudos e Pesquisas em Psicologia é licenciado sob uma Licença Creative Commons Atribuição-Não Comercial 3.0 Não Adaptada. 